\title{
REVIEW
}

\section{Mechanisms and innovations}

\section{Cardiac stimulation with high voltage discharge from stun guns}

\author{
Kumaraswamy Nanthakumar MD, Stephane Massé PEng MASc, Karthikeyan Umapathy PhD, \\ Paul Dorian MD, Elias Sevaptsidis, Menashe Waxman MD
}

\section{ABSTRACT}

The ability of an electrical discharge to stimulate the heart depends on the duration of the pulse, the voltage and the current density that reaches the heart. Stun guns deliver very short electrical pulses with minimal amount of current at high voltages. We discuss external stimulation of the heart by high voltage discharges and review studies that have evaluated the potential of stun guns to stimulate cardiac muscle. Despite theoretical analyses and animal studies which suggest that stun guns cannot and do not affect the heart, 3 independent investigators have shown cardiac stimulation by stun guns. Additional research studies involving people are needed to resolve the conflicting theoretical and experimental findings and to aid in the design of stun guns that are unable to stimulate the heart.

Une version française de ce résumé est disponible à l'adresse www.cmaj.ca/cgi/content/full/I78/II/I45I/DCI

CMAJ 2008;178(II):145I-7

Published at www.cmaj.ca on May I, 2008.

S tun guns are used to physically incapacitate a person by discharging controlled electrical energy into the body, thereby preventing effective muscular activity. Although the intention is to provide a safe means of subduing an uncooperative person, some studies have suggested that stun guns can stimulate cardiac muscle in addition to skeletal muscle, thus potentially promoting lethal cardiac arrhythmias. In this article, we review the scientific data about the direct effects of stun gun discharges on the heart during shock delivery. We discuss these issues in terms of electrostimulation and correlate them with theoretical and experimental data in the literature. We discuss the principles of cardiac stimulation from internal and external stimulation and examine the evidence for and against cardiac stimulation by stun gun discharges.

\section{Stun gun discharges}

An older method of stun guns application, called "drivestun," functioned like a cattle prod, which required direct contact between the electrodes of the source and the target. Stun guns are manufactured by different manufacturers (e.g., Aegis Industries, Stinger Systems, Taser International) and they operate under the general principle of high-voltage discharge with short pulse durations. However, their operation and shock charecteristics vary by manufacturer. For example, a recent model (X26, TASER International) feature 2 barbs attached to long copper wires that are rapidly propelled by compressed nitrogen and adhere to the target's skin or clothes. This stun gun generates an initial 3 microsecond electric pulse, which produces an electrical arc that creates a low-impedance pathway for electricity to reach the body with or without skin contact. ${ }^{1}$ The initial pulse is followed by longer pulses (roo microseconds) that deliver electrical energy to the target's body, which stimulates his or her nerves and skeletal muscles and results in incapacitation. This pattern is repeated at a frequency of I9 pulses per second. Incapacitation lasts for the duration of the discharge, which is typically 5 seconds but can be 15 seconds or longer if pressure on the trigger is maintained. The TASER $\mathrm{X}_{2} 6$ battery has the capacity to deliver up to 195 discharges of 5 seconds each, ${ }^{1}$ which corresponds to a duration of over 15 minutes. Other devices that have been studied include the $\mathrm{M}_{2} 6$ (TASER International) and the MK63 Trident (Aegis Industries), which is a stun baton. Each of these devices uses high frequency electrical pulses to incapacitate the target.

\section{Method of stunning}

Stunning can be attributed to I of 2 methods, which depend on the mode of application. In the "drive stun" method, the overwhelming factor is the creation of pain and hence compliance. The second method, in which electrodes are fired toward the target as projectiles, neuromuscular stimulation occurs over a larger area. In addition to pain, the device incapacitates the target by stimulating his or her motor nerves and muscles as well as sensory neurons. The duration and frequency of the pulses have been optimized to incapacitate the target, and different devices have varying effects depend-

From the University of Toronto (Nanthakumar, Massé, Umapathy, Dorian, Sevaptsidis, Waxman); the Division of Cardiology, Toronto General Hospital (Nanthakumar, Massé, Umapathy, Sevaptsidis, Waxman); and St. Michael's Hospital (Dorian), Toronto, Ont. 
ing on the frequency of stimulation and the shape of the electrical pulse.

\section{Electrical stimulation of the heart}

Since the early I $900 \mathrm{~s},{ }^{2,3}$ various equations have been proposed to describe the relation between the current and pulse duration required for electrostimulation of the heart. These formulas showed an inverse relation between the duration and the current of the stimulating pulse, which means that if the pulse duration is short, a higher current is required for stimulation. ${ }^{4}$

For an electrical pulse to stimulate the heart, it must depolarize the cardiac membrane below a certain level and the induced depolarization must be propagated throughout the heart. The duration and strength of the pulse must be sufficient to allow cell membranes to react and reach an excitation threshold above which activation is triggered. This activation produces a wave front resulting in mechanical contraction of the heart muscle. Shorter pulse durations require larger amounts of current or charge to stimulate the heart. Thus, one must consider whether a stun gun discharge, which is external to the heart, can deliver enough current to stimulate the heart. Below we discuss the external stimulation of the heart under other known circumstances and relate it to a typical electrical pulse generated by a stun gun.

\section{Effect of external electrical discharges on the heart}

The ability of external electrical discharges to alter the internal electrical activity of the heart (e.g., to induce ventricular fibrillation) has long been recognized. ${ }^{5}$ Depending on the method of delivery and the amount, timing and location of the electrical discharge, an external discharge can produce a beat when one is absent, induce fatal cardiac arrhythmias or restore a normal heart beat to a heart in arrhythmia. ${ }^{6}$ The use of external electrical discharges to influence the heart has resulted in the development of external pacemakers (e.g., Zoll stimulator) and defibrillator devices to treat ventricular fibrillation. However, these discharges are delivered under controlled conditions at rates that are physiologic or that are delivered during the safe part of the cardiac cycle. High voltage discharges commonly occur in various forms, from electro- static discharge (most common) to electrocution or lightning strike (least common). Internal cardiac defibrillators also use high voltage pulses for terminating ventricular fibrillation. The relative values for voltage, current and energy for some common sources of high-voltage shocks, along with the most common type of stun gun in use, are shown in Table I .

The physiologic effects of shocks from these sources vary depending on the duration, frequency and energy of the discharges. Burn injuries are usually local and minor for most sources, except for line voltage if the body is in contact long enough for heat to accumulate (Joule effect). In contrast, the major consequence of a lightning strike is a phenomenon known as electroporation, which creates holes or pores in cell membranes. This disruption can wreak havoc on nerve and muscle tissues. In addition, secondary currents induced by the magnetic field generated by the large current of a lightning strike could be large enough to cause cardiac arrest and seizure. $^{7}$

In contrast, discharges from static electricity (electrostatic discharge) or from a stun gun involve a small amount of energy (less than I joule). In both cases, electricity travels to the skin through an arc that provides a low impedance path, allowing the current to flow between the source and the target. The amount of energy is too small to create burns or local electroporation. In the case of electrostatic discharge, a brief uncomfortable shock is felt, but it has no physiologic consequences. The pain is perceived through sensory nerves. The operation of most stun guns is thought to rely on their effect on motor function, and pain is a collateral, but intense, effect. The frequency and the shape of the pulses generated by stun guns are designed to incapacitate the target by electrically overwhelming his or her control of these muscles. Though the experimental evidence supporting these claims is not entirely clear, the net effect is that the target cannot control his or her skeletal muscles. This effect lasts for at least the duration of the discharge. In principle, these pulses are designed to act only on skeletal muscles and to not affect internal organs such as the heart.

\section{Evidence that stun guns cannot stimulate the heart}

Despite the fact that stun guns are widely used and that their practical safety is under scrutiny, the majority of these analy-

\begin{tabular}{lcccc}
\hline $\begin{array}{l}\text { Table 1: Approximate electrical characteristics of some common sources of } \\
\text { high-voltage shocks }\end{array}$ & \\
\hline Source & Peak voltage & Peak current & Duration & Energy \\
\hline Lightning & $1 \mathrm{GV}$ & $40 \mathrm{kA}$ & $0.12 \mathrm{~ms}$ & $500 \mathrm{MJ}$ \\
\hline $\begin{array}{l}\text { Internal defibrillator } \\
\text { Electrostatic discharge }\end{array}$ & $750 \mathrm{~V}$ & $4-20 \mathrm{~A}$ & $5-20 \mathrm{~ms}$ & $35 \mathrm{~J}$ \\
\hline Shock from line voltage & $15-20 \mathrm{KV}$ & $30 \mathrm{~A}$ & $0.1 \mu \mathrm{s}$ & $60 \mathrm{~mJ}$ \\
$\begin{array}{l}\text { Shock from } \\
\text { electroconvulsive therapy }\end{array}$ & $120 \mathrm{~V}$ & $80-100 \mathrm{~mA}$ & $<1 \mathrm{~s}$ & $10 \mathrm{~J}$ \\
\hline TASER X26 & $450 \mathrm{~V}$ & $900 \mathrm{~mA}$ & $<1 \mathrm{~s}$ & $20 \mathrm{~J}$ \\
\hline
\end{tabular}


ses are theoretical in nature..$^{8-12}$ These theoretical analyses suggest that stun guns cannot deliver the amount of energy required to stimulate the heart or cause ventricular fibrillation. Most theoretical studies base their arguments on the following principles: only a small portion ( $4 \%-10 \%)$ of the current that reaches the chest will affect the heart ${ }^{13}$ and the time constant of the cardiac cell membrane is much longer than the pulse duration generated by stun guns. According to the law of electrostimulation and given the electrical characteristics of stun gun pulses and cardiac cells, cardiac electrostimulation should not occur during a stun gun shock. ${ }^{8}$ These analyses support the claim that electrical pulses generated by stun guns are designed to specifically target skeletal muscle, which has a much smaller time constant (i.e., refractoriness) compared with cardiac cells.

Experimental studies that support the claim that stun guns do not stimulate the heart base their arguments on conservative device settings and experimental designs that often do not reflect a clinically relevant or "worst case" scenario. The studies by Lakkireddy and colleagues ${ }^{14}$ and McDaniel and colleagues, ${ }^{15}$ both involving swine, used a modified stun gun for which the output power could be controlled, allowing the authors to specify a safety margin for the device and to demonstrate that it could not induce ventricular fibrillation. McDaniel and colleagues used arterial blood pressure tracing, which showed no perturbations during discharge from the stun gun simulator. ${ }^{15}$ However, intracardiac electrograms from the study by Lakkireddy and colleagues showed that the pulses did influence heart rate during shock delivery if the barbs were located such that they formed a vector crossing the heart. ${ }^{14}$ In contrast, the $\mathrm{MK}_{3} 3$ stun baton in the "drive stun" mode applied to the anterior thigh ${ }^{16}$ or tho$\operatorname{rax}^{17}$ of Yucatan miniature pigs did not induce acute arrhythmias. The authors of both studies attributed their findings of a lack of cardiac stimulation to possible differences in electrode spacing, proprietary waveform or power generated by the device.

Other studies ${ }^{18,19}$ have been performed using healthy volunteers (police officers). Each volunteer received a single 5 second stun gun pulse to his or her back. This does not reflect the common scenario, in which multiple, prolonged shocks are delivered with the possibility of the barbs landing near the thorax. These studies recorded electrocardiogram findings before and after, but not during, the stun gun discharge. This, however, does not rule out the possibility of disturbances in the rhythm during the discharge owing to the artifacts in recorded electrocardiograms during the discharge. These limitations prevented the researchers from observing transient changes in heart rhythm during discharges. The immediate recordings after the discharges ${ }^{18}$ showed shortening and lengthening of QT complexes without assigning any significance to these changes.

Stun gun discharges have been recorded in the field and there have been no claims of deaths medically attributed to these discharges. ${ }^{20}$ These recordings were made immediately after, but not during, the discharge. Although this does not affect the claim of no related deaths, these studies cannot verify whether the heart was stimulated during discharge. In cases of recorded deaths, the mode of death had never been established, though a state of "excited delirium" has been reported. ${ }^{21}$ However, excited delirium has not been listed as a cause of sudden cardiac death in the arrhythmia literature.

\section{Evidence that stun guns can stimulate the heart}

Deaths have occurred shortly after stun gun discharges. ${ }^{22}$ However, association alone does not prove causality. The possible mechanisms of short-term or immediate-term cardiac effects relate to the stimulation of the heart or induction of ventricular fibrillation. Stimulation of the heart is a separate issue compared with induction of arrhythmia, as stimulation may happen only during discharge and may not be evident even immediately following the discharge. In contrast, induction of arrhythmia may relate to stimulation of the heart because, depending on pre-existing defects (e.g., a previous heart attack, drug intoxication), each person's heart may have a different susceptibility to life-threatening arrhythmia during stimulation. Podgorski and colleagues ${ }^{23}$ found that the direct application of an older version of a stun gun to a pig heart, which was exposed but covered by a towel, produced stimulation of the heart.

Because the theory of electrical stimulation suggests that stun gun discharges should not stimulate the heart, we tested the hypothesis using a closed-chest in vivo animal model. ${ }^{24} \mathrm{~A}$ unique feature of our study was that real stun guns were used and operated by qualified law enforcement personnel, which simulated real-world conditions. Two different models (TASER X 26 and M26) that deliver different pulse waveforms were used on an anesthetized pig. Recording the electrical activity of the heart is challenging, because the acquisition system is usually completely saturated by the electromagnetic interference generated by the stun gun discharge. However, we found that the pig's arterial blood pressure was occasionally abruptly lost during stimulation. To further verify that this blood pressure modulation was not a recording artifact, we opened the artery to air and found that the pumping of blood stopped during the discharge of the stun gun. This made us suspicious that either an arrhythmia was being induced or the heart was being stimulated so rapidly that it was not capable of producing pulsatile pressure. To test this, we shielded our mapping system and recorded the electrical activity during discharge.

We studied a total of 150 discharges in 6 pigs. ${ }^{24}$ Of these, 74 of these discharges resulted in stimulation of the myocardium, as documented electrical capture (a provoked response in the myocardium) (mean ventricular rate during stimulation and capture, 324 [standard error 66 ] beats/min) (Figure I). Of the 94 discharges across the heart, 74 stimulated the myocardium. We took care to ensure that the gun barbs did not pierce deep into the tissue. We also placed the barbs such that they were oriented across the heart, simulating the worst case scenario of creating a current vector that directly passes through the heart. If these barbs were placed away from the chest and across the abdomen, none of the 56 discharges across the abdomen stimulated the heart 
(Figure 2), suggesting that the location of the barbs had a crucial influence on stimulating the heart. We also observed that the waveform (pulse shape) produced by the device affected stimulation, because when we used a different model of the stun gun (TASER M26), we observed a lower incidence of cardiac stimulation.

In addition, we simulated an excited state infusing pigs with epinephrine, which renders the myocardium more excitable and prone to arrhythmias. Of 16 discharges, there were 13 episodes of myocardial stimulation, of which I induced ventricular fibrillation and I caused ventricular tachycardia. In contrast, another study, ${ }^{14}$ which simulated an excited stated by infusing cocaine into pigs, did not report induction of ventricular fibrillation during discharge. The main conclusions of this study was that stun gun use in the presence of cocaine does not increase the chance of arrhythmia. However, this study used a waveform simulator, not an actual stun gun, and although ventricular fibrillation was not induced, there was stimulation of the heart.

Three different studies involving pigs, I of which was performed by us, have shown that a stun gun discharge can stimulate the heart. ${ }^{14,24,25}$ In particular, I study ${ }^{25}$ reported the deaths of 2 animals caused by ventricular fibrillation immediately after the stun gun discharge. This study also re- ported severe metabolic and respiratory acidosis caused by discharge. This suggests that sufficient current density was produced by the stun gun to stimulate the heart, which according to theory should not occur. A potential explanation of why, despite the theory, stimulation was observed is that there were metallic objects (e.g., catheter or pacemaker leads) inside the heart, which probably carried currents induced by the electromagnetic interference generated during the shock. One could argue that these currents could instead be the primary source of heart stimulation. Because capture could only be observed using intracardiac electrograms, this remains speculative. The fact that in our study we did not observe capture when the stun gun shocks were administered away from the chest suggests that the dart locations play a more important role in stimulation than the presence of metallic objects in the heart. In addition, in our study, we removed all electrical catheters from the heart and still observed the cessation of arterial pumping during discharge. We also confirmed that even without catheters in the heart, stun gun discharges on the chest can stimulate the heart and, at the least, can result in a loss of blood pressure during discharge.

Indeed, a human's chest is different from that of a pig, and there may be differences in electrophysiology between human

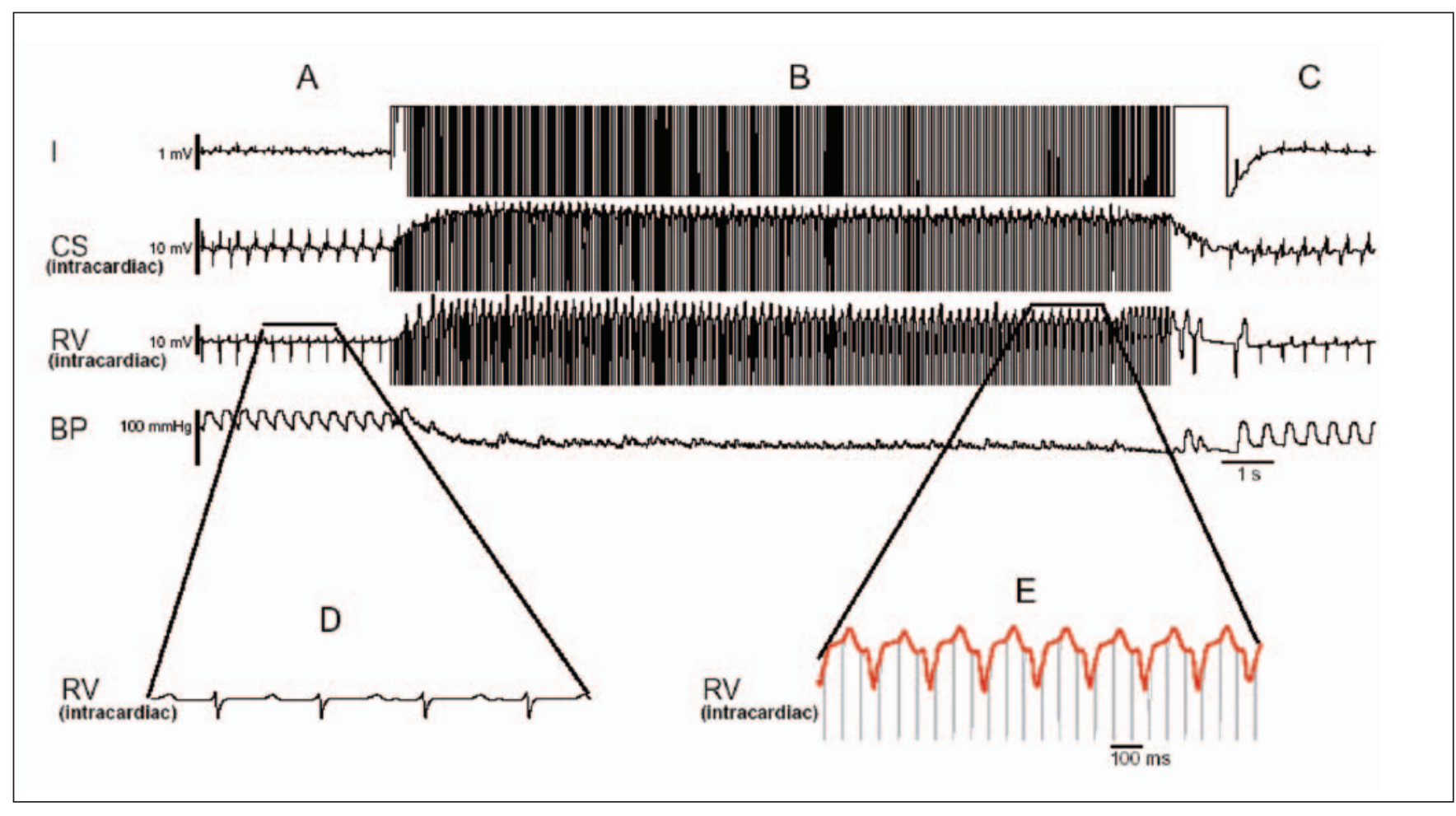

Figure 1: Cardiac stimulation and hypotension from a stun gun discharge. Note the corruption of the surface electrocardiographic leads in panel B and the electrical activity of the intracardiac electrograms. After stun gun discharge, a spontaneous and immediate return of regular sinus rhythm and blood pressure occurs (panel C). Panel D and E show magnified intracardiac electrograms of similar duration. It is evident in panel $E$ that the rate is much faster and the rhythm is wider than in panel $D$. The morphology of the tachycardia in panel $E$ is wider than the morphology in panel D. There is a constant stun gun stimulus artifact to electrogram duration as illustrated in panel $\mathrm{E}$, with every fourth stun gun discharge resulting in stimulation of the heart. Note the loss of blood pressure during the stimulation and the recovery of blood pressure once the discharge is completed. Reproduced with permission from Elsevier (Nanthakumar et al ${ }^{24}$ ). Note: $\mathrm{CS}=$ coronary sinus, $\mathrm{RV}=$ right ventricular, $\mathrm{BP}=$ blood pressure. 
and pig hearts. One should be prudent in extrapolating data from animals to humans because of this fact. The corollary, though, is that most of the basic mechanistic concepts in cardiac fibrillation and defibrillation are derived from animal studies, not humans. In addition, the safety margins for energy of stun gun discharge established by manufacturers were derived from animal models. ${ }^{15}$

Researchers from San Francisco recently published the case of a patient with a pacemaker who received a stun gun shock. ${ }^{26}$ They observed that discharges from the stun gun provoked a response in the myocardium (Figure 3). It is unknown if this would have occurred without the presence of pacemaker wires, although without these wires, verifying the presence of cardiac capture would not have been possible. In addition, John Webster's research group reported in a conference abstract that stun gun discharges can stimulate the heart. ${ }^{27}$ Although published theoretical analyses about stun gun safety have scientific merit, we should be aware that theories are only as good as the assumptions and conditions defined based on available data or knowledge.

\section{Explaining the discrepancies between theory and observation}

Why have 3 independent groups of investigators reported in peer-reviewed journals that cardiac stimulation can occur when the theory says it cannot happen? Theoretical safety calculations may not hold true if the theory used to calculate the membrane time constant using external pacing parameters (i.e., with large pads that do not break the skin barrier, without rapid stimulation at high voltage) does not apply to stun gun stimulation across the chest wall. Although the membrane constant is usually considered an intrinsic property of cardiac muscle, various studies have measured time constants during human trans-chest pacing from 0.5 milliseconds to I.I milliseconds. ${ }^{28}$ However, another study with direct pacing on dog myocardium reported an average value of 2.4 milliseconds ${ }^{29}$ suggesting that the time constant is actually a characteristic of not only the cell membrane but also the stimulator, and the size and the position of the electrode used. This suggests a large variability over the population; thus, an identical pulse with a specific duration and strength could have different stimulation effects on different people.

Over the last century, various studies have been performed on the strength-duration relation of electric impulses and their effect on cardiac stimulation. From some of these studies, it is evident that the assumptions made about membrane time constants and contact electrode sizes strongly influence the outcome. ${ }^{30}$ Typically, electrodes in contact within the myocardium may stimulate with 50 milliamperes when the current is injected over a period of 50 microseconds. However, shorter pulse durations would require a larger amount of current to stimulate the heart. There is a possibility of inducing a lethal cardiac arrhythmias when factors (e.g., strength, duration and frequency of the electric pulses; membrane time constant; contact impedance; and timing of elec-

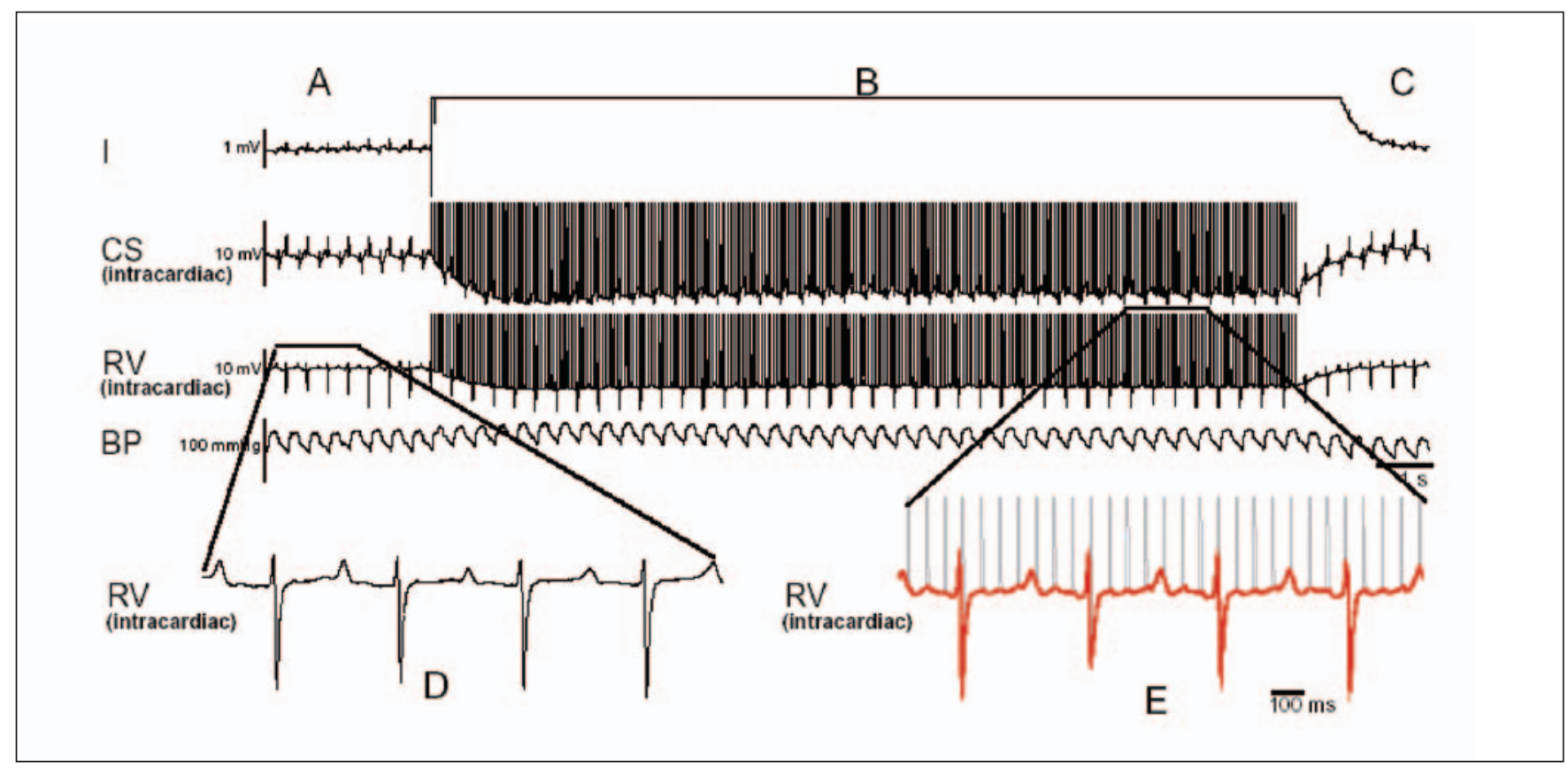

Figure 2: A typical episode of a stun gun shock across the abdomen (nonthoracic vector) that does not result in stimulation of the myocardium. The surface electrocardiogram lead 1, intracardiac electrograms from the coronary sinus, the right ventricle apex and blood pressure in the descending aorta are shown. Panel A illustrates the regular rhythm before the discharge, which is very similar to the rhythm and rate in panel $\mathrm{C}$. The intracardiac electrograms, as illustrated in panels $\mathrm{D}$ and $\mathrm{E}$, do not show any significant change in rate morphology and are not phase-locked (no temporal relation between stimuli and the electrogram) with the stun gun discharge. Note also the lack of perturbation of blood pressure during the discharge. Reproduced with permission from Elsevier (Nanthakumar et $\left.\mathrm{al}^{24}\right)$. Note: $\mathrm{CS}=$ coronary sinus, $\mathrm{RV}=$ right ventricular, $\mathrm{BP}=$ blood pressure. 


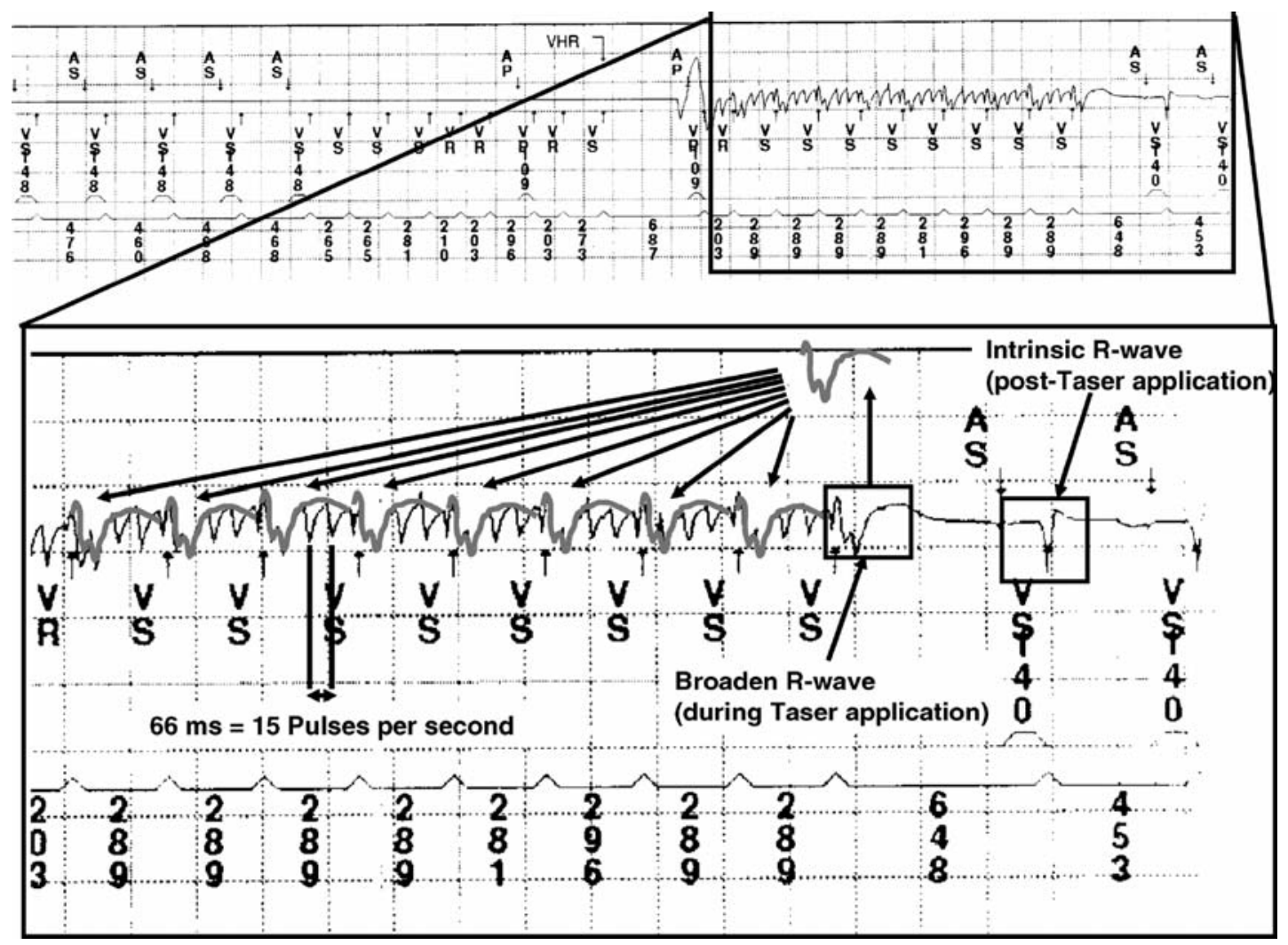

Figure 3: Magnified summed intracardiac electrograms from a patient's internal pacemaker log during stun gun discharge. Cardiac capture is shown by the high-rate ventricular sensing (cycle length $203-289$ milliseconds); the cyclic, low-frequency modulation of high-frequency noise (stun gun pulses) during ventricular sensing; a single, long ventricular interval ( 648 milliseconds) after the energy stops; and postdischarge resumption of atrial and ventricular sensing at a rate similar to predischarge cardiac rate. The high-frequency pulses (15 pps, 66 milliseconds) are labelled on the tracing. The intracardiac electrograms from the last sensed ventricular event during stun gun application are superimposed on each prior ventricular sensed event, showing that the disruption of the high-frequency stun gun signal is consistent with modulation of the signal by a repeating $\mathrm{R}$ wave with morphology different than the intrinsic $\mathrm{R}$ wave (right side of the image). Reproduced with permission from Blackwell (Cao et $\mathrm{al}^{26}$ ).

trical discharge) favour triggering the heart during a vulnerable period of the cardiac cycle.

\section{Knowledge gaps}

Although there have been deaths reported following stun gun discharges, this appears to be rare. In addition, some animal studies suggest that stun gun shocks may have cardiovascular effects. Whether the reported deaths were related to the external shocks is unknown. It is also unknown whether cardiac stimulation occurs only during discharge. The observational studies involving human volunteers thus far could be considered phase I studies because they relate mainly to tolerability and do not prove the safety of the devices. It is very important that tolerability should not be misconstrued as safety. The largest knowledge gap is the lack of appropriate studies involving humans to establish the safety margins for stun gun shock energies when the vector of discharge is across the heart.
The effects of potential modifying factors such as sex, body mass, cardiac and noncardiac diseases, alcohol, medications and psychotropic drugs also need to be evaluated. It is evident that psychotropic drugs such as cocaine heighten the sympathetic state in animal studies. ${ }^{31,32}$ The effect of these drugs and their influence on human autonomic physiology during stun gun discharges is an important aspect that needs urgent evaluation.

\section{Conclusions}

Despite many studies suggesting that stun guns do not affect the heart, the evidence and studies presented in this review suggest that, in some circumstances, stun guns may stimulate the heart while discharges are being applied. However, there is no conclusive evidence to show whether stun gun stimulation (under certain electrophysiological conditions) can result in cardiac arrhythmias late after stun gun discharge. In our view, it is inappropriate to conclude that stun 
gun discharges cannot lead to adverse cardiac consequences in all real world settings.

We believe that the findings that stun gun discharges are able, under specific circumstances, to stimulate the heart should be taken into account in future studies involving people. Whether stun guns can stimulate the heart can only be established if one can record electrical activity in the heart during a discharge, especially when the vector of discharge is directed across the heart. Additional research studies involving people will help to resolve the conflicting theoretical and experimental findings, and they could lead to the design of devices with electrical pulses that cannot stimulate the heart.

This article has been peer reviewed.

Competing interests: None declared.

Contributors: All of the authors contributed to the intellectual development and critical revision of the manuscript. All of the authors approved the version accepted for publication.

Acknowledgements: Dr. Nanthakumar is supported by the ClinicianScientist program of the Canadian Institutes of Health Research.

\section{REFERENCES}

I. TASER law enforcement catalog. Scottsdale (AZ): Taser International; 2006. Issue $2, \mathrm{p} .7$.

2. Weiss G. Sur la possibilité de rendre comparable entre eux les appareils servant à l'excitation électrique. Arch Ital Biol I9or;35:413-46.

3. Lapicque L. Considérations préalables sur la nature du phénomène par leque l'électricité excite les nerfs. JPhysiol Pathol Génér 1907;9:565-78.

4. Blair HA. The intensity-time relations for stimulation with electric currents. J Gen Physiol I932;15:709-29, 731-55

5. Hoffa M, Ludwig C. Einigeneue versuche über herzebewegung. $Z$ Rationelle Me. I850;9:107-44.

6. Wiggers CJ. The mechanism and nature of ventricular fibrillation. Am Heart I940;20:399-4I2.

7. Lee RC, Zhang D, Hannig J. Biophysical injury mechanisms in electrical shock trauma. Annu Rev Biomed Eng 2000;2:477-509.

8. Ideker RE, Dosdall DJ. Can the direct cardiac effects of the electric pulses generated by the TASER X 26 cause immediate or delayed sudden cardiac arrest in normal adults? FAm J Forensic Med Pathol 2007;28:195-201.

9. Stratbucker RA, Kroll MW, Mcdaniel W., et al. Cardiac current density distribution by electrical pulses from TASER devices. Institute of Electrical and Electronics Engineers International Conference of the Engineering in Medicine and Biology Society 2006; 6305-6307.

Io. Panescu D, Kroll MW, Efimov I, et al. Finite element modeling of electric field effects of TASER devices on nerve and muscle. Conference proceedings of the Institute of Electrical and Electronic Engineers; 2006 Aug 30-Sept 3; New York (NY) New York (NY): Engineering in Medicine and Biology Society; 2006. p. 1277-9.

II. Kroll MW. Crafting the perfect shock. IEEE Spectrum 2007;44:27-3I.

I2. Holden SJ, Sheridan RD, Coffey TJ, et al. Electromagnetic modelling of current flow in the heart from TASER devices and the risk of cardiac dysrhythmias. Phys Med Biol 2007;52:7193-209.

13. Lermann BB, Deale OC. Relation between transcardiac and transthoracic curren during defibrillation in humans. Circ Res i990;67:1420-6.

I4. Lakkireddy D, Wallick D, Ryschon $\mathrm{K}$, et al. Effects of cocaine intoxication on the threshold for stun gun induction of ventricular fibrillation. J Am Coll Cardiol 2006; 48:805-II.

I5. McDaniel WC, Stratbucker RA, Nerheim M, et al. Cardiac safety of neuromuscular incapacitating defensive devices. Pacing Clin Electrophysiol 2005;28:S284-7.

I6. Valentino DJ, Walter RJ, Dennis AJ, et al. Neuromuscular effects of stun device discharges. J Surg Res 2007;143:78-87.

I7. Valentino DJ, Walter RJ, Nagy K, et al. Repeated thoracic discharges from a stun device. J Trauma 2007;62:1134-42.

I8. Levine SD, Sloane CM, Chan TC, et al. Cardiac monitoring of human subjects exposed to the TASER. J Emerg Med 2007;33:II3-7.

19. Vilke GM, Sloane CM, Levine SD, et al. Twelve-lead electrocardiogram monitoring of subjects before and after voluntary exposure to the Taser X26. Am J Emerg Med 2008;26:I-4.

20. Wilkinson D. Scientific development branch further evaluation of TASER devices.
Home Office Police Scientific Development Branch 2005

2I. Strote J, Range HH. Taser use in restraint-related deaths. Prehosp Emerg Care 2006;10:447-50.

22. Kim PJ, Franklin WH. Ventricular fibrillation after stun gun discharge. NEJM 2005 353:958-9

23. Roy OZ, Podgorski AS. Tests on a shocking device - the stun gun. Med Biol Eng Comput $1989 ; 27: 445-8$

24. Nanthakumar K, Billingsley IM, Masse S, et al. Cardiac electrophysiological consequences of neuromuscular incapacitating device discharges. J Am Coll Cardiol 2006;48:798-804.

25. Dennis AJ, Valentino DJ, Walter RJ, et al. Acute effects of TASER $X_{2} 6$ discharges in a swine model. J Trauma 2007;63:58I-90.

26. Cao M, Shinbane JS, Gillberg JM, et al. Taser-induced rapid ventricular myocardial capture demonstrated by pacemaker intracardiac electrograms. J Cardiovasc Electrophysiol 2007; 18:876-9.

27. Will JA, Wu JY, Sun H, et al. Can TASERS directly cause ventricular fibrillation? 2006 Experimental Biology Meeting, April I-5 2006; Abstract \# 327

28. Irnich W. The chronaxie time and its practical importance. Pacing Clin Electrophysiol I980;3:292-301.

29. Pearce JA, Bourland JD, Neilsen E, et al. Myocardial stimulation with ultrashort duration current pulses. Pacing Clin Electrophysiol ig82;5:52-8

30. Geddes LA, Babbs CF, Voorhees WD III, et al. Choice of the optimum pulse duration for precordial cardiac pacing: A theoretical study. Pacing Clin Electrophysio I985;8:862-9.

3I. Shannon RP, Mathier MA, Shen Y.Role of cardiac nerves in the cardiovascular response to cocaine in conscious dogs. Circulation 2001;103:1674-80.

32. Keller DJ, Todd GL. Acute cardiotoxic effects of cocaine and a hyperadrenergic state in anesthetized dogs. Int J Cardiol I994;44:19-28.

Correspondence to: Dr. Kumaraswamy Nanthakumar, Division of Cardiology, Toronto General Hospital, GW 3-522, I50 Gerrard St. W, Toronto ON $\mathrm{M}_{5} \mathrm{G}_{2} \mathrm{C}_{4}$

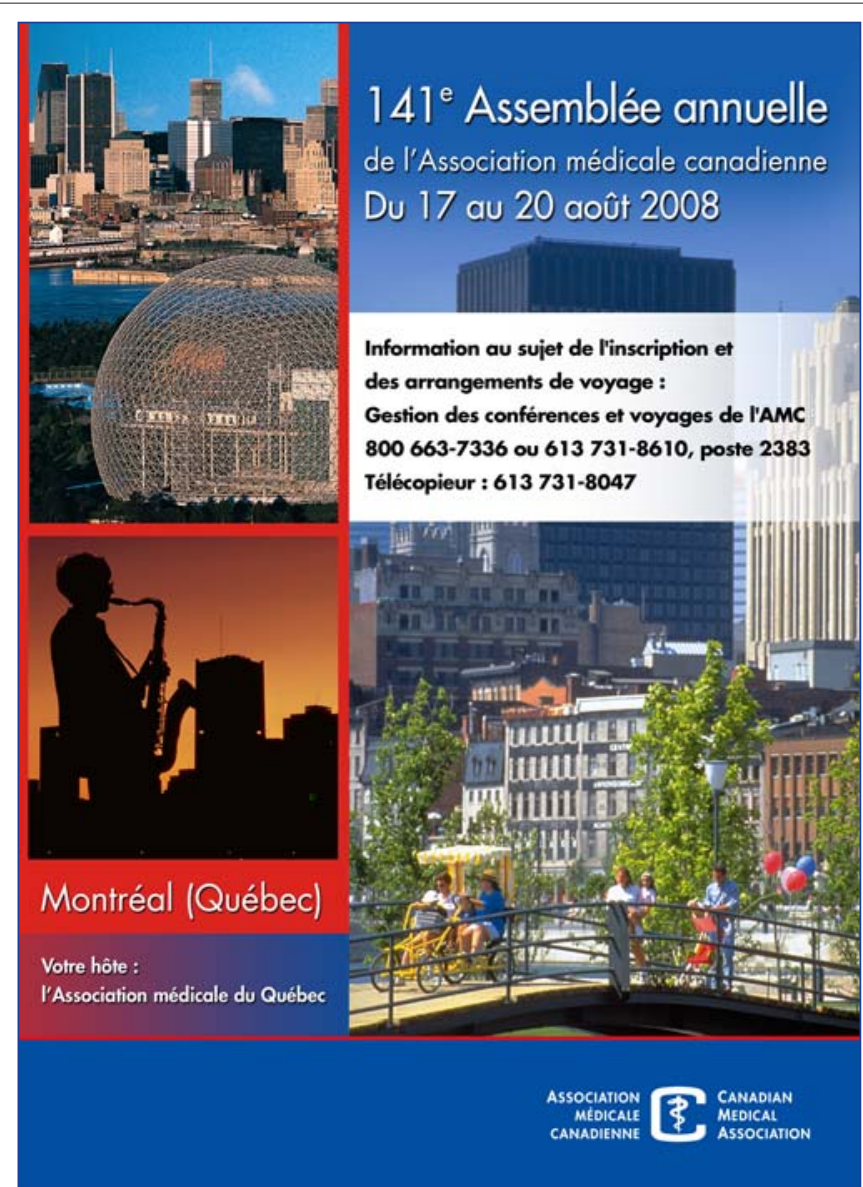

